\title{
Stability Boundaries and Sufficient Stability Conditions for Stably Stratified, Monotonic Shear Flows
}

\author{
Makoto Hirota \\ Institute of Fluid Science, Tohoku University, Sendai, Miyagi 980-8577, Japan \\ Philip J. Morrison \\ Department of Physics and Institute for Fusion Studies, University of Texas at Austin, \\ Austin, Texas 78712, USA
}

\begin{abstract}
Linear stability of inviscid, parallel, and stably stratified shear flow is studied under the assumption of smooth strictly monotonic profiles of shear flow and density, so that the local Richardson number is positive everywhere. The marginally unstable modes are systematically found by solving a one-parameter family of regular Sturm-Liouville problems, which can determine the stability boundaries more efficiently than solving the Taylor-Goldstein equation directly. By arguing for the non-existence of a marginally unstable mode, we derive new sufficient conditions for stability, which generalize the Rayleigh-Fjørtoft criterion for unstratified shear flows.
\end{abstract}

Keywords: hydrodynamic stability, stratified shear flow, Sturm-Liouville problem

\section{Introduction}

Instabilities of stratified shear flows are important for understanding not only geophysical phenomena but also fundamental mechanisms of flow instabilities. The couplings among vortical waves and gravity waves, supported by the choice 5 of background basic state, give rise to various types of instabilities named after Kelvin, Helmholtz, Holmboe, and Taylor (see the reviews [1, 2]). By replacing gravity by centrifugal force, the analogous mechanism further triggers instabil-

Preprint submitted to Physics Letters A

March 28, 2016

(C) 2016. This manuscript version is made available under the Elsevier user license http://www.elsevier.com/open-access/userlicense/1.0/ 
ities of swirling flows and vortices. The above instabilities have been described most clearly by assuming staircase profiles of the ambient vorticity and density, which enables one to interpret the instabilities in terms of the couplings of a finite number of discrete eigenmodes [1, 2]. Slightly-smoothed staircase profiles are studied approximately by using matched asymptotic expansions [3].

However, it is generally difficult to predict and interpret the instabilities of more realistic smooth profiles because the stable disturbances occupy a continuous spectrum [4, 5, even for a fixed streamwise wavenumber and for a wallbounded domain (which was explicitly pointed out by Case [6]). Since the corresponding eigenvalue problem, called the Taylor-Goldstein equation, is nonself-adjoint and singular, the accurate computation of the stability boundary is often hard both analytically and numerically. The classical Miles-Howard criterion [5, 7] states that the stratified shear flow is stable if the local Richardson number $J$ is greater than $1 / 4$ everywhere. Although many theoretical investigations have been made concerning various situations where $J \leq 1 / 4$ somewhere (see [8, 9, 10, and references therein), a general stability criterion for smooth profiles does not appear to be in the literature. However, a generalized version of Rayleigh-Fjørtoft criterion is found when $0<J \leq 1 / 4$ everywhere in Ref. [11.

In this letter, we will present an efficient method for finding marginally unstable modes in the case of $J>0$ everywhere. Following this method, we will further obtain new sufficient conditions for stability.

\section{Search for Marginally Unstable Modes}

We consider the linear stability of parallel shear flow $\boldsymbol{U}=(0, U(x))$ in an inviscid, incompressible fluid of variable density $\rho(x)$ on a domain $(x, y)$ bounded by two walls at $x= \pm L$, where the gravitational acceleration $g$ acts in the $-x$ direction. By introducing the stream function of the disturbance as $\phi(x) e^{i k(y-C t)}$ with a complex phase speed $C \in \mathbb{C}$ and a (real) wavenumber $k>0$, stability is 35 governed by the Taylor-Goldstein (TG) equation:

$$
\phi^{\prime \prime}-k^{2} \phi+\frac{U^{\prime \prime}}{C-U} \phi+\frac{N^{2}}{(C-U)^{2}} \phi=0, \quad \phi( \pm L)=0,
$$


where the prime $\left({ }^{\prime}\right)$ indicates the $x$ derivative and the Boussinesq approximation $\left[\rho(x)=\rho_{0}+\delta \rho(x)\right.$ where $\left.\rho_{0} \gg|\delta \rho|\right]$ has been used, with $N=\sqrt{-g \rho^{\prime} / \rho_{0}}$ being the Brunt-Väisälä (or buoyancy) frequency. If this equation has a nontrivial solution for $C$ with a positive imaginary part, $\operatorname{Im} C>0$, the shear flow is 40 spectrally unstable. In what follows, we assume a strictly increasing shear flow and a stably stratified density, i.e.,

$$
U^{\prime}>0 \quad \text { and } \quad \rho^{\prime}<0 \quad \text { on }[-L, L]
$$

so that the local Richardson number $J=N^{2} / U^{2}$ is positive everywhere. (Strictly decreasing shear flows $U^{\prime}<0$ can be treated similarly by replacing $U$ by $-U$ in the TG equation. In fact, a flow $U$ is stable if and only if $-U$ is so.) When the 45 domain is unbounded $(L=\infty)$, we also assume that $U$ and $\rho$ approach bounded values at infinity.

Stability boundaries are often studied by searching for marginally unstable eigenmodes with $C=c+i 0=\lim _{\epsilon \rightarrow+0}(c+i \epsilon)($ where $c=\operatorname{Re} C \in \mathbb{R})$, which were called singular neutral modes by Miles [5] because the TG equation becomes singular at the critical layer $x_{c}$ satisfying $c=U\left(x_{c}\right)$. Miles proved that a singular neutral mode may exist only when $J\left(x_{c}\right) \leq 1 / 4$ and only in the form of either $\phi \propto \phi_{+}$or $\phi \propto \phi_{-}$(see the results VIII and IX of [5]), where

$$
\phi_{ \pm}=(c+i 0-U)^{1 / 2 \pm \nu_{c}} \varphi_{ \pm}(c, x)
$$

with the sign of $\nu_{c}=\sqrt{1 / 4-J\left(x_{c}\right)}$ delineating the two linearly independent solutions obtained by the Frobenius method, and $\varphi_{ \pm}$are real analytic functions satisfying $\varphi_{ \pm}\left(c, x_{c}\right) \neq 0$ as well as the boundary condition $\varphi_{ \pm}(c,-L)=$ $\varphi_{ \pm}(c, L)=0$. Specifically, the singularity of 3 has the following branch cuts extending to infinity,

$$
\begin{aligned}
& (c+i 0-U)^{1 / 2 \pm \nu_{c}} \\
& =|c-U|^{1 / 2 \pm \nu_{c}} \exp \left[i \pi\left(\frac{1}{2} \pm \nu_{c}\right) H\left(x_{c}-x\right)\right],
\end{aligned}
$$

where $H(x)$ is the Heaviside function. Thus, the existence of the domain $D_{w}=$ $\{x \in[-L, L]: J(x) \leq 1 / 4\}$ is necessary for instability, in that the singular 
neutral modes must have the critical layers on it. Since $0 \leq \nu_{c}<1 / 2$ for $x_{c} \in D_{w}$, we will refer to the less singular mode $\phi_{+}$as the "small" neutral mode, and $\phi_{-}$as the "large" neutral mode (following the terminology of ideal MHD stability theory [12]) 1]. Notice that these two types of neutral modes degenerate to $(c+i 0-U)^{1 / 2} \varphi(c, x)$ only when $J\left(x_{c}\right)=1 / 4$ (which usually occurs when $x_{c}$ is an endpoint of $\left.D_{w}\right)$. For later use, we also define $\check{D}_{w}=\{x \in$ $[-L, L]: J(x)<1 / 4\}$ by omitting such the endpoints.

In order to find these singular neutral modes efficiently, we first apply a transformation $\psi=(C-U)^{-1 / 2+\nu_{c}} \phi$ to the TG equation [13] and obtain, for $C=c+i 0$,

$$
\left(P \psi^{\prime}\right)^{\prime}-\left(k^{2}+Q\right) P \psi=0, \quad \psi( \pm L)=0
$$

70 where

$$
\begin{aligned}
& P\left(x, x_{c}\right)=|U-c|^{1-2 \nu_{c}} \exp \left[i \pi\left(1-2 \nu_{c}\right) H\left(x_{c}-x\right)\right], \\
& Q\left(x, x_{c}\right)=\left(\frac{1}{2}+\nu_{c}\right) \frac{U^{\prime \prime}}{U-c}-U^{\prime 2} \frac{J-J\left(x_{c}\right)}{(U-c)^{2}} .
\end{aligned}
$$

Because $|P|^{-1},|P|$ and $|Q P|$ are integrable functions for given $x_{c} \in \check{D}_{w}$, this transformed equation is considered to be a regular Sturm-Liouville equation [16, [17, and hence $\psi$ and $P \psi^{\prime}$ are continuous including the point $x=x_{c}$. Moreover, we note from (3) that the small and large neutral modes, respectively, satisfy $75 \psi_{+}\left(x_{c}\right)=0$ and $\left(P \psi_{-}^{\prime}\right)\left(x_{c}\right)=0$ at the critical layer $x_{c} \in \check{D}_{w}$. Base on this fact, we divide the domain into $\left[-L, x_{c}\right]$ and $\left[x_{c}, L\right]$, and seek the small and large solutions on each side as follows. By introducing the operator,

$$
\mathcal{E}\left(x_{c}, \lambda\right) \psi:=\left(|P| \psi^{\prime}\right)^{\prime}-(\lambda+Q)|P| \psi,
$$

that depends on the parameter $\lambda=k^{2}>0$, we solve

$$
\begin{array}{r}
\mathcal{E}\left(x_{c}, \lambda_{L+}\right) \psi=0, \psi(-L)=0, \psi\left(x_{c}\right)=0, \\
\mathcal{E}\left(x_{c}, \lambda_{L-}\right) \psi=0, \psi(-L)=0,\left(|P| \psi^{\prime}\right)\left(x_{c}\right)=0,
\end{array}
$$

\footnotetext{
${ }^{1}$ Actually, there is yet another type of neutral modes that may exist on the ranges $c \leq$ $U(-L)$ and $U(L) \leq c$ as the gravity waves. But, they are always stable according to Howard's semi-circle theorem [7].
} 
on $\left[-L, x_{c}\right]$ and

$$
\begin{array}{r}
\mathcal{E}\left(x_{c}, \lambda_{R+}\right) \psi=0, \psi(L)=0, \psi\left(x_{c}\right)=0, \\
\mathcal{E}\left(x_{c}, \lambda_{R-}\right) \psi=0, \psi(L)=0,\left(|P| \psi^{\prime}\right)\left(x_{c}\right)=0,
\end{array}
$$

80 either Dirichlet or Neumann boundary condition at $x=x_{c}$, Sturm's oscillation theorem [17] guarantees that the eigenvalues can be ordered as follows.

$$
\begin{aligned}
& \infty>\lambda_{L-}^{(1)}>\lambda_{L+}^{(1)}>\lambda_{L-}^{(2)}>\lambda_{L+}^{(2)}>\lambda_{L-}^{(3)}>\cdots \rightarrow-\infty, \\
& \infty>\lambda_{R-}^{(1)}>\lambda_{R+}^{(1)}>\lambda_{R-}^{(2)}>\lambda_{R+}^{(2)}>\lambda_{R-}^{(3)}>\cdots \rightarrow-\infty,
\end{aligned}
$$

where the superscript $(n)$ indicates the $n$th largest eigenvalue. Here, only a finite number of positive eigenvalues is of our interest (since $\lambda=k^{2}>0$ ) and $D_{w} \backslash \check{D}_{w}$ (namely, $J\left(x_{c}\right)=1 / 4$ ), the degeneracy, $\lambda_{L-}^{(n)}=\lambda_{L+}^{(n)}$ and $\lambda_{R-}^{(n)}=\lambda_{R+}^{(n)}$ $(n=1,2, \ldots)$, must occur. If the matching condition $\lambda_{L-}=\lambda_{R-}>0$ between the left and right domains is satisfied for a specific $x_{c} \in D_{w}$, there is a large neutral mode $\psi_{-}$for such $c=U\left(x_{c}\right)$ and $k=\sqrt{\lambda_{L-}}=\sqrt{\lambda_{R-}}$. Similarly, there exists a small neutral mode $\psi_{+}$if $\lambda_{L+}=\lambda_{R+}>0$ occurs for a specific $x_{c} \in D_{w}$.

Thus, all neutral modes can be found systematically in the parameter space $\left(x_{c}, \lambda\right) \in D_{w} \times[0, \infty]$. In numerical computation, positive eigenvalues $(\lambda>0)$ of the regular Sturm-Liouville problems (7)-(10) are scanned for every $x_{c} \in$ $D_{w} \subset[-L, L]$ and, only if they exist, the aforementioned crossing among them 95 is searched. Various iteration methods (such as the shooting method and the conjugate gradient method) are useful for obtaining the real eigenvalues of (7)(10) in descending order and their continuous dependence on the scan parameter $x_{c}$. For the purpose of determining the stability, this numerical approach is more efficient than solving for complex eigenvalues of the TG equation for each $k$.

(i) $U=\tanh (10 x), N^{2}=22$,

(ii) $U=x, N^{2}=0.1+10 x^{2}$, 

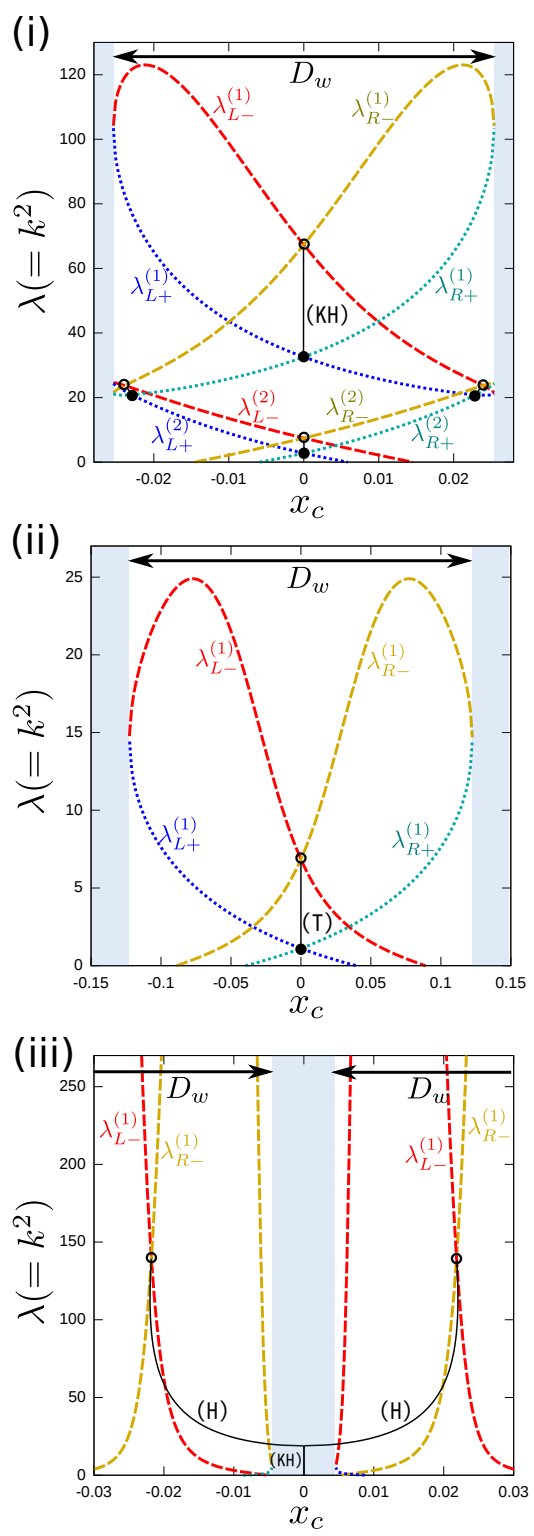

Figure 1: Eigenvalues $\lambda_{L-}, \lambda_{L+}, \lambda_{R-}$ and $\lambda_{R+}$ of (7)-10 scanned over $x_{c} \in D_{w}$ (dashed and dotted color lines) that are calculated numerically for the examples of unstable flows (i)(iii). Large and small neutral modes $(C=c+i 0)$, respectively, exist at the parameters of open and closed dots; $c=U\left(x_{c}\right)$ and $k=\sqrt{\lambda}$. The real parts $(c=\operatorname{Re} C)$ of unstable eigenvalues for each $k$ are independently calculated and plotted as black solid lines through the mapping $x_{c}=U^{-1}(c)$. 
(iii) $U=\tanh (10 x), N^{2}=30 /\left(1+10^{4} x^{2}\right)$,

on the common domain $[-L, L]=[-1,1]$; the basic states (i)-(iii) are primarily

105 ities, respectively. The results are shown in Fig. 1(i)-(iii), where the shaded areas indicate the exterior of $D_{w}$. Several large and small neutral modes are found, respectively, as indicated by the open and filled circle symbols in Fig. 1 . We have also searched for neighboring unstable eigenvalues $(\operatorname{Im} C>0)$ by the

shooting method and confirmed that unstable modes indeed exist between these neutral modes. The black solid lines indicate the locus of $x_{c}=U^{-1}(c)$ calculated from the real parts $c=\operatorname{Re} C$ of the unstable eigenvalues. For the case (i), the stratification induces three more unstable modes in addition to the $\mathrm{KH}$ instability that originally exists in the mixing layer. As exemplified by these modes as well as the Taylor instability (ii), increasing the stratification (or $N^{2}$ ) generally tends to spawn more unstable modes, whose eigenfunctions are more oscillatory (i.e., $\lambda_{L \pm}^{(n)}$ and $\lambda_{R \pm}^{(n)}$ with larger $n$ show up on the positive side). At the same time, however, the width of $D_{w}$ tends to be narrowed by increasing $N^{2}$, which in turn contributes to stabilization. In the case (iii), $D_{w}$ is divided into two parts by the sharply peaked profile of $N^{2}$ (or density jump) and the KH mode is split into the Holmboe modes. Here, it should be noted that the unstable modes may exist outside $D_{w}$ while the neutral modes are forced to exist inside $D_{w}$.

\section{Sufficient Conditions for Stability}

125 Now, we recall that Howard [7] obtained the upper bound of $\operatorname{Im} C$ as $\operatorname{Im} C<$ $k^{-1} \max _{[-L, L]} \sqrt{U^{\prime 2} / 4-N^{2}}$, which implies $\operatorname{Im} C \rightarrow 0$ as $k \rightarrow \infty$. On the other hand, we have shown the absence of neutral modes for sufficiently large wavenumber $k$ satisfying either $k^{2}>\lambda_{L-}^{(1)}$ or $k^{2}>\lambda_{R-}^{(1)}$ for all $x_{c} \in D_{w}$, which proves stability at $k \rightarrow \infty$. By noting that the eigenvalues must move continuously on the upper half plane of $C$ as the parameter $k^{2}$ changes, unstable eigenvalues remain absent as $k^{2}$ decreases from $\infty$ and such an eigenvalue can 
only emerge via a marginally unstable eigenvalue $C=c+i 0$. Therefore, the absence of neutral modes for sufficiently large $k$ proves the absence of any unstable mode for the same range of $k$. Since the largest eigenvalues, $\lambda_{L-}^{(1)}$ and $\lambda_{R-}^{(1)}$, of the Sturm-Liouville problem are easily calculated by the variational method, we can derive a stability condition in the following form.

Criterion 1. The flow (2) is spectrally stable for the wavenumber $k$ that satisfies either

$$
\begin{gathered}
k^{2}>-\min _{\psi(-L)=0} \frac{\int_{-L}^{x_{c}}|P|\left(\psi^{\prime 2}+Q \psi^{2}\right) d x}{\int_{-L}^{x_{c}}|P| \psi^{2} d x}, \\
\text { or } \quad k^{2}>-\min _{\psi(L)=0} \frac{\int_{x_{c}}^{L}|P|\left(\psi^{\prime 2}+Q \psi^{2}\right) d x}{\int_{x_{c}}^{L}|P| \psi^{2} d x},
\end{gathered}
$$

for all $x_{c} \in D_{w}$.

140 Suppose that we have computed the right hand sides of 11 and 12 (namely, $\lambda_{L-}^{(1)}$ and $\left.\lambda_{R-}^{(1)}\right)$ for all $x_{c} \in D_{w}$ and found that they are negative on subsets $D_{w 1} \subset D_{w}$ and $D_{w 2} \subset D_{w}$, respectively. If $D_{w}=D_{w 1} \cup D_{w 2}$, the flow is spectrally stable for all $k$. Note that either $D_{w 1}$ or $D_{w 2}$ may be the null set and then 111 or 12 will be not applicable.

If $Q\left(x, x_{c}\right) \geq 0$ both on $\left[-L, x_{c}\right]$ for all $x_{c} \in D_{w 1}$ and on $\left[x_{c}, L\right]$ for all $x_{c} \in D_{w 2}$, Criterion 1 immediately proves stability. This observation enables us to derive further stability conditions as follows. Let $a \in[-L, L]$ be the rightmost point in $D_{w 1}$, i.e., $a=\max D_{w 1}$. By using the inequality

$$
\frac{J\left(x_{c}\right)-J(x)}{U\left(x_{c}\right)-U(x)} \geq \min _{[-L, a]} \frac{J^{\prime}}{U^{\prime}} \quad \text { for } x \leq x_{c} \leq a,
$$

we get

$$
Q\left(x, x_{c}\right) \geq \frac{U^{\prime 2}}{c-U}\left[-\left(\frac{1}{2}+\nu_{c}\right) \frac{U^{\prime \prime}}{U^{\prime 2}}+\min _{[-L, a]} \frac{J^{\prime}}{U^{\prime}}\right],
$$

on $\left[-L, x_{c}\right] \subset[-L, a]$. The requirement $Q\left(x, x_{c}\right) \geq 0$ on $\left[-L, x_{c}\right]$ for all $x_{c} \in D_{w 1}$ (for which $0 \leq \nu_{c}<1 / 2$ ) leads to 15 below. A similar inequality can also be derived for $\left[x_{c}, L\right] \subset[b, L]$ in terms of the leftmost point $b$ in $D_{w 2}$. Thus, we obtain the following stability criterion: 
Criterion 2. The flow (2) is spectrally stable if there exist $a, b \in[-L, L]$ such that

$$
\begin{gathered}
\max _{\substack{[-L, a] \\
\sigma=1 / 2,1}}\left(\sigma \frac{U^{\prime \prime}}{U^{\prime 2}}\right) \leq \min _{[-L, a]}\left(\frac{J^{\prime}}{U^{\prime}}\right), \\
\min _{\substack{[b, L] \\
\sigma=1 / 2,1}}\left(\sigma \frac{U^{\prime \prime}}{U^{\prime 2}}\right) \geq \max _{[b, L]}\left(\frac{J^{\prime}}{U^{\prime}}\right),
\end{gathered}
$$

and $D_{w} \subset[-L, a] \cup[b, L]$ hold, where (15) or (16) may be omitted when $a=-L$ or $b=L$, respectively.

We remark that, in [15], for example, we should adopt $\sigma=1 / 2$ if $\max _{[-L, a]} U^{\prime \prime}<$ 0 , or $\sigma=1$ otherwise.

From Criterion 2, it is straightforward to obtain the next criterion:

Criterion 3. The flow (2) is spectrally stable if there exist $a, b \in[-L, L]$ such that

$$
\begin{gathered}
U^{\prime \prime} \leq 0 \text { and } J^{\prime} \geq 0 \text { on }[-L, a], \\
U^{\prime \prime} \geq 0 \text { and } J^{\prime} \leq 0 \text { on }[b, L],
\end{gathered}
$$

and $D_{w} \subset[-L, a] \cup[b, L]$ hold, where (17) or 18 may be omitted when $a=-L$ or $b=L$, respectively.

Recall that unstratified shear flows with no inflection point $\left(U^{\prime \prime} \neq 0\right)$ were shown to be stable by Rayleigh [14, and even when a monotonic shear flow $\left(U^{\prime}>0\right)$ has one inflection point $U^{\prime \prime}\left(x_{I}\right)=0$, it is still stable if $U^{\prime \prime}<0$ on $\left[-L, x_{I}\right]$ and $U^{\prime \prime}>0$ on $\left[x_{I}, L\right]$ according to Fjørtoft [15]. Criterion 3 asserts that these flows remain stable even with the effect of stratification, if the additional condition on the sign of $J^{\prime}$ is satisfied (where $a=b=x_{I}$ is chosen for the Fjørtoft case).

Since $J^{\prime} \lessgtr 0$ is equivalent to $\left(N^{2}\right)^{\prime} / N^{2}=\rho^{\prime \prime} / \rho^{\prime} \lessgtr U^{\prime \prime} / U^{\prime}$ by definition, the case of $N^{2} \equiv$ const. (or $\rho^{\prime \prime} \equiv 0$ ) automatically satisfies the condition on $J^{\prime}$ of Criterion 3. On the contrary, if $J$ has a local minimum in the interior of $[-L, L]$ (i.e., somewhere except at the wall $x= \pm L$ ) and its value is below $1 / 4$, Criterion 1753 is violated and such a stratification can destabilize an otherwise stable shear 

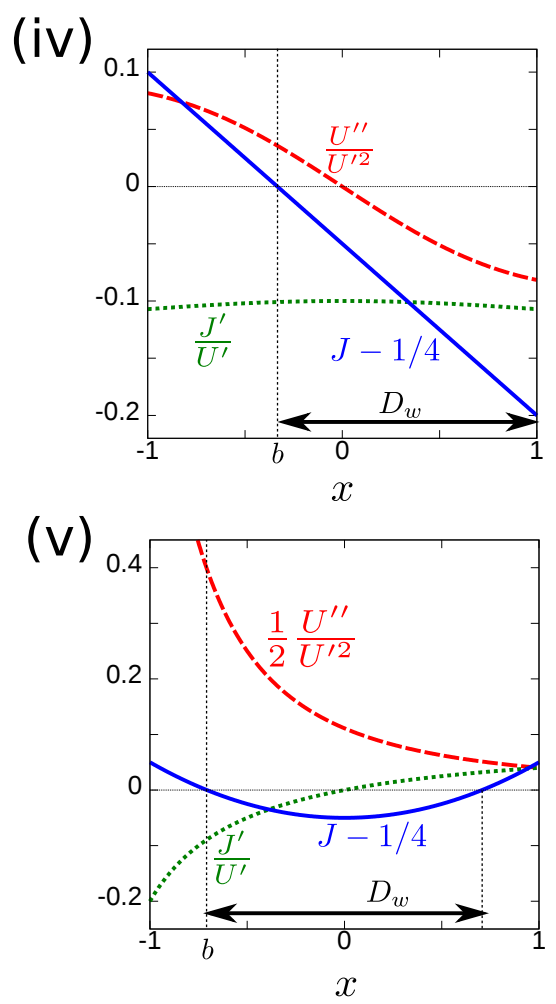

Figure 2: Examples of stable flows (iv) and (v) found by Criterion 2

flow, like the Taylor instability of Fig. 1(ii). Criterion 3 is also violated by a $U^{\prime}$ that has a local maximum (i.e., a local maximum of vorticity) in the interior of $D_{w}$, which can cause $\mathrm{KH}$ instability like the example of Fig. 11(i).

The more detailed Criterion 2 tells us that this destabilization effect of $U^{\prime \prime}$ 180 (or $J^{\prime}$ ) may be suppressed by the stabilization effect of $J^{\prime}$ (or $U^{\prime \prime}$ ). For example, the flows,

(iv) $U=\arctan (x / 2)+x, J=(1-3 x / 4) / 5$,

(v) $U=(x+3 / 2)^{2}, J=\left(x^{2}+2\right) / 10$,

on $[-L, L]=[-1,1]$ are found to be stable by Criterion 2. For the case (iv), the 185 sign of $U^{\prime \prime}$ violates the Rayleigh-Fjørtoft criterion and $U^{\prime}$ has a maximum in $D_{w}$, but the stratification $J$ contributes to stabilization so that the inequality 


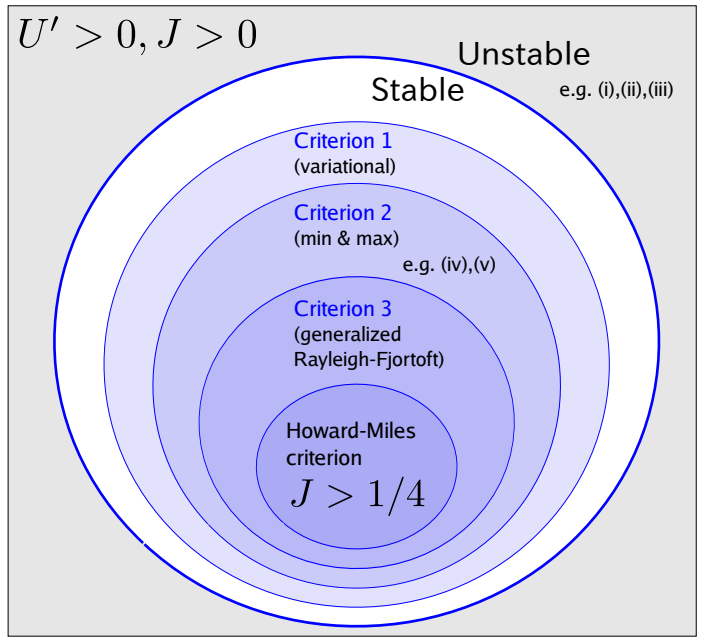

Figure 3: Venn diagram of sufficient stability criteria

(16) with $D_{w}=[b, L]$ holds, as shown in Fig. 2 Conversely, for the case (v), $J$ has a minimum that is below $1 / 4$, but $U^{\prime \prime}>0$ is large enough to satisfy 16 with $D_{w} \subset[b, L]$.

\section{Concluding Remarks}

In summary, by using the method developed in this letter, we can efficiently find singular neutral modes that are embedded in the continuous spectrum. The unstable ranges of wavenumber are searched for by solving the regular SturmLiouville problems (7)-10 for each $x_{c} \in D_{w}$, which requires less labor than solving the TG equation (i.e., a non-self-adjoint eigenvalue problem) for each $k>$ 0 directly. With the help of numerical computation, this method will be useful for determining stability boundaries for various flows and understanding their instability mechanisms. Based on this method, we have derived new stability criteria (Criteria 1,2, and 3) as extensions of the Rayleigh-Fjørtoft criterion to stratified flows, which are of course improvements of the Howard-Miles criterion since the local Richardson number is allowed to be less than 1/4 (see Fig. 3).

We expect that the present approach could yields stability boundaries of 
non-monotonic shear flows and locally unstratified (or constant) densities with additional or different treatments for the singularity. A few specific examples were investigated in earlier works 18, 9, 10, which in particular suggests that the singularity at the critical layer should be replaced by that of Rayleigh's equation when $N^{2}\left(x_{c}\right)=0$. By making use of the abundant knowledge about Rayleigh's equation [19, 20, more detailed stability criteria tailored for these applications will be discussed elsewhere.

This work was supported by JSPS KAKENHI Grant Number 25800308 and by JSPS Strategic Young Researcher Overseas Visits Program for Accelerating Brain Circulation \# 55053270. PJM was supported by DOE Office of Fusion Energy Sciences, under DE-FG02-04ER-54742.

\section{References}

[1] L. N. Howard, S. A. Maslowe, Boundary-layer Meteorology, 4, 511 (1973).

[2] J. R. Carpenter, E. W. Tedford, E. Heifetz, G. A. Lawrence, Appl. Mech. Rev. 64, 061001 (2012).

[3] N. J. Balmforth, A. Roy, C. P. Caulfield, J. Fluid Mech. 694, 292 (2012).

[4] A. Eliassen, E. Høiland, E. Riis, Two-Dimensional Perturbation of a Flow with Constant Shear of a Stratified Fluid, Institute for Weather and Climate Research, Norwegian Academy of Sciences and Letter, Publ. no. 1 (The Institute of Theoretical Astrophysics, Oslo, 1953).

[5] J. W. Miles, J. Fluid Mech., 10, 496 (1961).

[6] K. M. Case, Phys. Fluids, 3, 143 (1960).

225 [7] L. N. Howard, J. Fluid Mech., 10, 509 (1961).

[8] A. Alexakis, Phys. Fluids, 17, 084103 (2005). A. Alexakis, Phys. Fluids, 19, 054105 (2007). 
[9] S. M. Churilov, J. Fluid Mech. 539, 25 (2005). S. M. Churilov, J. Fluid Mech. 617, 301 (2008). S. M. Churilov, Geophys. Astrophys. Fluid Dyn. (to be published).

[10] T. Rees, A. Monahan, J. Atmos. Oceanic Technol. 31, 2795 (2014).

[11] A. Banerjee, arXiv:physics/0510114v1 [physics.flu-dyn] (2005).

[12] W. A. Newcomb, Ann. Phys. 10, 232 (1960)

[13] A similar transformation is used in J. W. Miles, J. Fluid Mech., 16209 (1963).

[14] Lord Rayleigh, Proc. Lond. Math. Soc. 11, 57 (1880).

[15] R. Fjørtoft, Geofys. Publ. 17, 1 (1950).

[16] H. D. Niessen, A. Zettl, Proc. Lond. Math. Soc. 64545 (1992).

[17] A. Zettl, Sturm-Liouville Theory, volume 121 of Mathematical surveys and 240 monographs (American Mathematical Society, Providence, 2005).

[18] H.E. Huppert, J. Fluid Mech. 57, 361 (1973)

[19] N. J. Balmforth, P. J. Morrison, Stud. Appl. Math. 102, 309 (1999).

[20] M. Hirota, P. J. Morrison, Y. Hattori, Proc. R. Soc. A 470, 20140322 (2014). 